Available online at GSC Online Press Directory

GSC Biological and Pharmaceutical Sciences

e-ISSN: 2581-3250, CODEN (USA): GBPSC2

Journal homepage: https://www.gsconlinepress.com/journals/gscbps

(REVIEW ARTICLE)

\title{
Integrative molecular navigator in preventable diseases: Pulmonary arterial hypertension (PAH) and rare diseases
}

\author{
Mukesh Kataria, Bonnie Welch, Diane E Heck and Hong Duck Kim * \\ Department of Public Health, School of Health Sciences and Practice, New York Medical College, Valhalla NY.
}

Publication history: Received on 31 August 2020; revised on 27 September 2020; accepted on 28 September 2020

Article DOI: https://doi.org/10.30574/gscbps.2020.12.3.0285

\begin{abstract}
Pulmonary arterial hypertension (PAH) is a debilitating lung condition for which there has been no cure that leads to complications on the right side of the heart. This disorder is currently distinguished from other conditions of the right ventricle by a DNA biorepository, invasive hemodynamics, echocardiography, genotype-tissue expression, imaging, and other diagnostic tools. Present treatment strategies include mainly medications and oxygen therapy choices. Advanced omics developments, including next generational genetic analysis, massively parallel gene-editing, metabolomics, and pharmacogenomics have significantly improved the volume of information that can be analyzed effectively in individuals with pulmonary arterial high blood pressure among other chronic illnesses. Emerging molecular-driven and gene targeting-driven evidence shed light on a new era of innovation as advanced clinical technology for providing patients quality of care in case of systemic arterial morbidity recognition, early diagnosis, therapeutic validation, and safety, including precision treatments based on patients' genomic data pool obtained through genetic imprinting or genetic mapping.
\end{abstract}

Keywords: Pulmonary arterial hypertension (PAH) metabolomics; Polymorphism; Pharmacogenomics; Drug safety; Gene editing

\section{Introduction}

Emerging molecular based detection techniques, like multifold omics, can be employed more effectively to assign patients care towards their own potentially targeted treatments. This review focuses on the importance of awareness of genetic identification and management of pulmonary arterial hypertension (PAH). Advantages and shortcomings of existing omics innovations are addressed. Additionally, we examine, how specific technological innovations could be employed to potentially enhance treatment of systemic arterial infection more efficiently when treating with actual and future medications. Creating new knowledge of disease progression and mechanisms can influence future handling success or deficiency [1].

Bioinformatic approaches to evaluate a large amount of biomolecular information are continually changing. They also present significant challenges to the understanding of possible omics results in systemic arterial disorder along with a small portion of individuals examined and a probably high false alarm frequency [2]. With more data integration such as supplemental specific and proven drug response concepts, precision medicine can contribute as a critical element of the therapeutic practice of pulmonary hypertension $(\mathrm{PH})$ clients and the community encompassed to $\mathrm{PH}$ associated secondary health complications.

\footnotetext{
${ }^{*}$ Corresponding author: Hong Duck Kim

Department of Public Health, School of Health Sciences and Practice, New York Medical College, Valhalla NY. 
Despite significant advancements in the last several decades, pulmonary arterial depression remains a severe cause of injury and death. The risk condition predominantly presents, for example increased arterial blood pressure or pulmonary hypertension (PH) likely the systemic arterial disorder often involves pulmonary embolism, including congenital anomalies [1]. PH has also been segmented throughout the existing classification system into diseases with underlying connective heart conditions, including persistent thrombotic pulmonary hypertension disorders wherein $\mathrm{PH}$ is known to be a supplementary syndrome. For example, left ventricular pressure increases systemic vascular pressures and consequently, idiopathic pulmonary stress. However, if the spinal canal stress were eliminated, the PH might decline, logically. Likewise, cellular contents in lung issues, including coronary artery disease, are correlated with PH rarely, but pathophysiologic observations may not reliably show coronary artery vasculopathy.

Most of these disorders potentially expose to pulmonary artery dysfunction and eventually lead to death. New therapies are emerging, making enhanced diagnostic and therapeutic strategies potentially extremely medically meaningful. Many types of $\mathrm{PH}$, including persistent thrombotic respiratory muscles (CTEPH), have unique surgical procedures treatments. Still, the bulk of PH and PAH have no treatment apart from palliative treatment. Further exacerbating this category of diseases has been the absence of appropriate diagnostic information. Lung biopsy is usually used to indicate which might be associated with elevated risk in persons with PH and other types of systemic arterial disorder, making treatment based on medical conditions, radiographic observations, and intrusive hemodynamics. These phenotypic outcomes can be beneficial for physicians to determine whether the disease has progressed but carry a significant risk of possibly ignoring a treatable disease, including PAH or CTEPH, when identifying specific types of pulmonary hypertension. PAH-focused treatment has also been identified as perhaps affecting individuals with non-PAH $\mathrm{PH}$, however a clinical trend of merely addressing all arterial conditions is particularly detrimental. In brief, the sector does require better management methods and much more effective processes by which to classify clinicians who react to a condition. The purpose of quantitative and qualitative risk assessment relies on the identification of how omics can be applied to control morbidities in the diagnostic process which do not have an available cure. This review will conduct research into how PAH can be identified and prevented using omics. The aim of omics is to identify risk through a stepwise process with different molecular targets using a multi-fold strategy. Advanced omics such as lipidomic and pharmacogenomic can be applied in an attempt to control morbidity rates which did not show improved recovery rates previously. This approach will use qualitative analysis where observation design will be used to analyze bio-samples to identify risk morbidities which has no cure in animal subjects but can be prevented using molecular prediction approaches like advanced omics. Recent trends in medical science and bioengineering methods are now accessible to visualize molecular structure and it's dysconnectivity which led to the systemic arterial disorder. They carry considerable promise for improving diagnostic performance and enhanced handling of all types of cardia artery conditions. The core word "omics," derived from genomic, transcriptomics, or proteomics, apply to the area of PAH research in combination with advanced omics field including pharmacogenomics or lipidomic.

Broadly, omics implies the evaluation of the wide range of target molecules such as enzymes, receptors, transmitter, or single gene mutation which can lead to a medical illness or occurrence. While early research in omics involved analyses of big data in genomics, transcriptomics, metaproteomic along with bioinformatics, particularly the latest research has incorporated other areas such as urology (radionics), including molecular genetics (cell bionics). The utilizations of functional genomics, including biotechnology in the systemic arterial condition, is most known. Up to date, new studies have brought attention to what other omics can be used to recognize and efficiently manage the systemic arterial condition. PH identification is an essential step to predict potential risk early to care in patients with respect to determinant factors such as common physiological and metabolic shapes and patterns that correspond with similar pathological processes and therapy reactions in drugs. Even though changes have been identified, the assessment of PH remains focused on diagnostic features. Another significant exception would be hereditary PAH wherein hereditary defects, including those in transcription factors protein receptors expressed (BMPR2) and other abnormalities, have been well-described and can be utilized to assist evaluation of drug therapy in the clinic using translational indicator [3].

Although disease category utilizing conventional imaging approaches is usually very helpful in determining responsiveness to $\mathrm{PAH}$-directed treatments, several clinicians have identified characteristics with numerous forms between PH, as such-called "mashed" or "blended" PH.[4] An illustration of a typical person with cumulative category 1 and 2 PH seems to be the 75-year older adult, a sibling through genetic heritage to a person with hereditary PAH, who appears to have healthy breathing via chest examination. No scientific proof-of-evidence in severe thrombotic PH by airflow respiration lung test, as well as a right-sided heart laparoscopy showing a coronary artery wedge stress of 20 $\mathrm{mmHg}$ as well as arterial blood pressure of 95/50 $\mathrm{mmHg}$ with just standard blood pressure. The increased idiopathic pulmonary differential stress indicates second group $\mathrm{PH}$, although a person has a high diastolic differential pressure, indicating the presence of respiratory vasculopathy. 
In previous, it was reported a comprehensive analysis of 1,416 serum compounds in individuals with PAH versus healthy individuals utilizing ultra-performance liquid chromatography-mass spectroscopy [1]. Therefore, they included congenital and genetic inheritance individuals with $\mathrm{PAH}$, maintaining a reasonably homogeneous population relative to clients on safe and infection. Using an objective analytical method, they established compounds that differentiated PAH from conditions and discovered 20 compounds that distinguish PAH from stable measures and infection control mechanisms after correction for multiple variables. The overview of these compounds by the system illustrates improvements in fatty acid, cytidine, and fructose, including cholesterol profile. Researchers also established an eigenvalues rating utilizing seven compounds to distinguish PAH from restrictions on safe and illness [5]. Taken together, these data indicate that metabolomics could be employed to identify observable, physiological variations among PAH patients and healthy controls clients, and could point to key pathways for developing or sustaining PAH [6]. The research outcome has used using genetic engineering to explain how genomes trends in individuals with $\mathrm{PH}$ are much more comparable to PAH than those in second group PH with independent endothelial lining PH. Furthermore, other study identified characteristics of PH characterized by left heart disease throughout the study of three treatment populations and demonstrated that the combined post-and pre-capillary $\mathrm{PH}(\mathrm{Cpc}-\mathrm{PH})$ is distinctive and relatively young-age compared to isolated post-capillary $\mathrm{PH}$ (Ipc-PH) clinicians with much more extreme respiratory ischemia. Employing a biorepository of Genetic material containing existing knowledge on nucleotide sequence variations connected to anonymized clinical evidence, Cpc-PH developed specific genetic mutation variations which have become more specific to PAH compared to clients having Ipc-PH and had been considered to be relevant to PAH mechanisms including plasma membrane and insulin sensitivity [7]. These results indicate that genetic variation variations can explain both the pathogenesis of the systemic arterial disorder and the identification of pulmonary arterial pathophysiological processes and endophenotypes. Utilized similar techniques, it was examined and investigated further trends of expressions in the respiratory system, which is a highly relevant tissue phenotypic pattern for the pulmonary arterial disorder [8]. Pulmonary arterial hypertension (PAH) is a particular type of PH wherein the distal respiratory bronchioles produce occlusive defects, known as plexiform abnormalities, as well as many other modifications that typically result in altitudes of systemic vascular blockage with proper heart damage and death within 3-5 years if not handled adequately $[9,10]$.

\section{Conclusion}

In conclusion, although substantial improvement has been achieved in the treatment of Pulmonary Arterial Hypertension (PAH) in recent years, it remains cause of a significant mortal condition and demanded further research along with a contemporary analysis in pursuit of precision medicine. A considerable percentage of individuals diagnosed with PAH succumb within five years, which is equivalent to the introductory level of lung cancer recovery. At present, drug options are available with several type of treatments endorsed by the FDA across three broad groups including vasodilator, anti-proliferative agents, calcium channel blockers and others, but clinicians have a minimal ability to assess due to shortage of pharmacogenomics entity and its efficacy data which reflect balance of drug efficacy and toxicity among several class of drugs without personal genomics. This molecular omics approach could be advantageous prior to the decision process like safety and validation studies including lung toxicity which may lead to adverse effects of drugs, and increased therapy pressure under operational and genetic abnormality cases without any direct medical potential. In future studies, multi-omics approaches will play a key role in improvement quality of personal care by precision risk assessment and safety. Following the adaption of new platforms such as imaging and genomics data (e.g., whole genomics sequence) to define personalized single-nucleotide polymorphisms (SNPs) or family-oriented SNPs supplemented with biotechnology. The future will also be influenced by tools like CRISP or analyzing of small molecules like microRNA, blended with metabolomics and pharmacogenomics in the lung biopsy samples from patients or experimental models of PH and those who are suffering in Pulmonary arterial hypertension (PAH).

\section{Compliance with ethical standards}

\section{Acknowledgments}

This work was supported by the Student fund in School of Health Science and Practice and Institute of Public Health, New York Medical College.

\section{Disclosure of conflict of interest}

No conflict financial interests these authors declared. 


\section{References}

[1] Hemnes AR. Using omics to understand and treat pulmonary vascular disease. Frontiers in Medicine. 2018; 5, 157.

[2] Chen D, Zhao X, Sui Z, Niu H, Chen L, Hu C, Xuan Q, Hou X, Zhang R, Zhou L, Li Y, Yuan H, Zhang Y, Wu J, Zhang L, Wu R, Piao H-L, Xu G, Jia W. A multi-omics investigation of the molecular characteristics and classification of six metabolic syndrome relevant diseases. Theranostics. 2020; 10(5): 2029-2046.

[3] Teruel M, Chamberlain C, Alarcón-Riquelme ME. Omics studies: their use in diagnosis and reclassification of SLE and other systemic autoimmune diseases. Rheumatology. 2017; 56(suppl_1): i78-i87.

[4] Sun YV, Hu YJ. Integrative analysis of multi-omics data for discovery and functional studies of complex human diseases. In Advances in genetics. 2016; 93: 147-190.

[5] Straube J, Gorse AD, Huang BE, Lê Cao KA. A linear mixed model spline framework for analyzing time-course 'omics' data. PloS one. 2015; 10(8): e0134540.

[6] Hinterwirth H, Stegemann C, Mayr M. Lipidomics: the quest for molecular lipid biomarkers in cardiovascular disease. Circulation: Cardiovascular Genetics. 2014; 7(6): 941-954.

[7] Robinson JL, Nielsen J. Integrative analysis of personal omics data using biomolecular networks. Molecular BioSystems. 2016; 12(10): 2953-2964.

[8] Wang Q, Peng WX, Wang L, Ye L. Toward multiomics-based next-generation diagnostics for precision medicine. Personalized Medicine. 2019; 16(2): 157-170.

[9] Ovesná J, Slabý O, Toussaint O, Kodíček M, Maršík P, Pouchová V, Vaněk T. High throughput 'omics' approaches to assess the effects of phytochemicals in human health studies. British Journal of Nutrition. 2008; 99(E-S1): ES127-ES134.

[10] Diller GP, Gatzoulis MA. Pulmonary vascular disease in adults with congenital heart disease. Circulation. 2007; 115(8): 1039-1050. 